\title{
Archéopages
}

Archéopages

Archéologie et société

Hors-série 2 | 2010

Archéologie sans frontières

\section{Archéologie d'intervention autour de la Méditerranée}

Frank Braemer et Michel Gras

\section{(2) OpenEdition}

1 Journals

Édition électronique

URL : https://journals.openedition.org/archeopages/777

DOI : 10.4000/archeopages. 777

ISSN : 2269-9872

\section{Éditeur}

INRAP - Institut national de recherches archéologiques préventives

\section{Édition imprimée}

Date de publication : 1 octobre 2010

Pagination : 65-73

ISSN : $1622-8545$

\section{Référence électronique}

Frank Braemer et Michel Gras, «Archéologie d'intervention autour de la Méditerranée », Archéopages

[En ligne], Hors-série 2 I 2010, mis en ligne le 01 octobre 2010, consulté le 23 février 2023. URL : http:// journals.openedition.org/archeopages/777 ; DOI : https://doi.org/10.4000/archeopages.777

Tous droits réservés 


\section{Archéologie d'intervention autour de la Méditerranée}

\author{
Frank Braemer \\ CNRS \\ Michel Gras \\ École française de Rome
}

L 'organisation par les pays (principalement) occidentaux de missions archéologiques

dans des pays du pourtour méditerranéen a une longue histoire qui remonte au dernier tiers $\mathrm{du} \mathrm{XIX}^{\mathrm{e}}$ siècle. Si les études et fouilles sur un site forment l'essentiel des projets programmés et de longue durée, depuis les années 1960 une part non négligeable d'entre elles provient d'une demande des pays hôtes pour des sites «en péril ».

Cette demande crée une forme d'intervention archéologique dont il est utile de caractériser les modes de travail et l'évolution dans le panorama général de l'archéologie préventive ou de sauvetage.

Archéologie programmée à l'étranger. La plupart de ces missions se font à l'initiative des équipes de recherche qui négocient avec le pays dit « hôte» une « concession », généralement sur un site, et assurent entièrement l'organisation et le financement de leur opération de recherche. Des campagnes de terrain annuelles, d'une durée de quelques mois, conduites par des équipes réunissant chercheurs et professeurs, une équipe technique souvent réduite (topographe, architecte, dessinateur) et des étudiants confirmés permettent l'acquisition de données, qui sont entièrement mises en forme, traitées et publiées, par la même équipe, dans les pays demandeurs. Ces données primaires reviennent dans les pays hôtes sous la forme de rapports plus ou moins détaillés, accompagnés d'une documentation graphique et photographique, de listings d'objets, et de publications diverses. Ce modèle a un peu évolué au cours des 15 dernières années avec le développement de «missions conjointes» associant une équipe du pays hôte à l'équipe étrangère, correspondant, dans la réalité, à des niveaux de collaboration aux équilibres fort divers en matière de choix scientifiques et de conservation/traitement des données, et, encore plus, en matière de publications. Quelques chiffres suffisent à évaluer l'importance actuelle de cette forme d'archéologie autour de la Méditerranée : cinq pays européens (France, Allemagne, Royaume-Uni, Italie, Espagne) soutiennent annuellement plus de 320 projets de terrain autour de la Méditerranée. Les équipes qui réalisent ces projets regroupent entre 1 ooo et 1500 chercheurs, doctorants et techniciens, répartis sur l'ensemble des pays méditerranéens. Le budget annuel global de fonctionnement sur le terrain de ces projets, hors salaires, est au minimum de l'ordre de 8 millions d'euros.

Brève histoire de l'archéologie d'intervention en Méditerranée. C'est la " campagne de Nubie» lancée par l'Égypte sous l'égide de l'Unesco en 1960 dans le périmètre du haut barrage d'Assouan qui constitue le point de départ d'une nouvelle forme d'intervention archéologique. La demande explicite est formulée par un pays qui fait un appel d'offres international à financement, d'une part, et propose une collaboration scientifique, d'autre part, pour la réalisation d'opérations archéologiques dans le cadre de travaux d'aménagement de grande ampleur du territoire. Ce sont alors le calendrier de l'opération d'aménagement, sa limite géographique et la nature des travaux qui s'imposent aux équipes archéologiques: on est là clairement dans une pratique d'intervention préventive ou de sauvetage sur un mode qui a prévalu jusque dans les années 1990 dans les pays des grands fleuves du Proche-Orient, Turquie, Irak, Syrie et Égypte, et qui est encore en vigueur aujourd'hui, par exemple avec l'appel pour intervention sur le nouveau barrage syrien de Khanouqa sur l'Euphrate. Ces appels internationaux ont permis un élargissement notable du cercle des pays organisant une archéologie à l'étranger, ou un élargissement géographique des espaces traditionnels d'intervention de certains pays occidentaux. L'Espagne, la Pologne, les Pays-Bas, les pays scandinaves et le Japon ont développé à ces occasions leur action sur un terrain qui était jusque-là dominé par la France, l'Allemagne, le Royaume-Uni, les États-Unis et l'Italie. Ces campagnes ont également favorisé le développement et la capacité d'intervention des pays dans lesquels l'aménagement est réalisé : le nombre des opérations conduites par les services des Antiquités et les universités nationales a également connu un accroissement constant.

Sur le plan scientifique, il est certain que ces interventions ont renouvelé totalement notre vision de l'histoire de ces contrées et les problématiques de recherche par rapport à celles établies dans les années 1930 à 1960 autour de la fouille de quelques « grands» sites : le volume des corpus documentaires, le nombre de chercheurs impliqués, les surfaces fouillées ont fortement augmenté et des régions peu ou mal connues jusque-là ont pu être explorées. Mais l'organisation et les pratiques de recherche de terrain sont restées celles de la « mission archéologique à l'étranger » : seules les campagnes de terrain sont plus longues, avec des équipes parfois un peu plus professionnalisées. Il faut noter que ces interventions sur les grands barrages sont réalisées sur une longue durée, la dizaine d’années, sur des terrains souvent inoccupés. En conséquence, l'impératif d'urgence dans le temps et les contraintes techniques de l'environnement du chantier, qui imposent souvent une organisation différente du travail, ne sont pas les paramètrse majeurs de la réalisation de ces opérations.

Certains réaménagements urbains, ou bien des aménagements de grands sites en vue de leur exploitation touristique dans les années 1980 et 90 ont également donné lieu à des appels à collaboration internationale. Les expériences du centre-ville de Beyrouth, des parcs archéologiques 
de Jerash et de Petra en Jordanie ou de Carthage en Tunisie ont bien montré à la fois les limites de ce type d'organisation et introduit dans le paysage de lintervention des équipes plus professionnalisées: sur un terrain de surface réduite qui nécessite une coordination et un échange constant d'informations pour établir une conduite raisonnée des chantiers, le système des équipes autonomes scientifiquement, depuis l'opération de terrain jusqu'à sa publication, limite la communication qui permettrait la formulation journalière de choix et de priorités dans des perspectives scientifiques partagées, et sur la base d'une documentation commune. Le suivi sur le long terme, par une équipe étrangère, d'opérations d'aménagement de sites réalisées par les services archéologiques locaux (Bosra ou Palmyre en Syrie) relève de la même pratique.

Dans la plupart des cas, les données enregistrées sur le terrain sont traitées et publiées dans les pays d'origine des missions, ou, dans certains cas, par les instituts étrangers installés sur place. Le mode majeur de retour d'informations dans les pays hôtes est celui du colloque suivi des synthèses documentaires. Il n'y a, à notre connaissance, que la campagne de Nubie et les travaux à Carthage qui ont été suivis de la constitution de fonds documentaires dignes de ce nom : une partie significative des enregistrements de données primaires et des résultats scientifiques est conservée, dans le premier cas, en Égypte, au Centre d'étude et de documentation sur l'ancienne égypte (CEDAE), au sein du conseil suprême des Antiquités, grâce à une collaboration franco-égyptienne de très longue durée initiée en 1973 et, dans le second cas, en Tunisie, pour ce qui concerne la conservation de Carthage.

\section{Évolutions récentes de l'archéologie à}

l'étranger. C'est une autre logique de coopération qui a été suivie pour plusieurs opérations lancées au début des années 1990 après quelques cas expérimentaux (Karnak) : installation durable (dix à quinze ans minimum) d'une équipe conjointe sur place assurant la coordination générale des opérations archéologiques préalables aux aménagements de très grands monuments (citadelle de Damas), de secteurs urbains (murailles du Caire fatimide, Gaza), de zones d'aménagement touristique (Cumes, en Campanie) ou bien encore de villes entières (Alexandrie), puis gérant leur post-fouille sur place ainsi que la conservation des données et des objets, et assurant parfois le conseil en matière d'aménagement par les instituts et/ou au sein des services des Antiquités.

Cette archéologie d'intervention a fonctionné et trouvé sa justification morale et politique, ses modes de collaboration et ses financements en s'inspirant principalement de la convention de l'Unesco sur le patrimoine mondial (1972) : l'étude scientifique du Patrimoine commun de l'humanité et la diffusion des connaissances sur ce patrimoine sont les moteurs et le complément essentiel de sa préservation. La chaîne de production, de conservation et d'exploitation des connaissances est donc un continuum qu'il est impératif d'assurer.
Dans la plupart des cas, ce sont des institutions de recherche académique qui ont pris en charge cette fonction.

Il est remarquable de constater que ces formes d'intervention ont touché principalement des ensembles de sites menacés de disparition massive, visible et brutale (mise en eau des grands barrages), ou bien des sites majeurs (capitales antiques ou modernes, grands monuments). Mais les innombrables travaux d'aménagement, d'importance variable, routiers, agricoles, hydrauliques, urbains, qui se développent autour de la Méditerranée ne font pas l'objet de telles interventions. L'autoroute qui traverse le Maghreb de Sfax, en Tunisie, à Agadir, au Maroc, sur plus de $2500 \mathrm{~km}$, les nombreux aménagements de lacs collinaires dans tous les pays méditerranéens, les lignes de métros urbains, les très grandes opérations de mise en forme des champs en vue de l'agriculture mécanisée, etc., n'ont donné lieu à aucun appel international et les chantiers préventifs ou de sauvetage à l'initiative des autorités locales se comptent presque sur les doigts de la main.

\section{Évolution du milieu archéologique après} la convention de Malte. Les années 1990 et 2000 sont marquées en Europe par la mise en application des recommandations de la convention de Malte du Conseil de l'Europe. De nouvelles formes de rapports apparaissent avec le développement des structures nationales en Europe (organisme public de recherche en archéologie préventive, services de collectivités territoriales ou intégrés au ministère de la Culture, ou sociétés d'archéologie « commerciale»). L'initiative et la direction scientifique sont clairement du ressort des autorités du pays demandeur qui peuvent agir sur un appel d'offres commerciales dans les pays de la convention de Malte : c'est l'ouverture à la concurrence et à «l'archéologie commerciale », qui a commencé en France ces trois ou quatre dernières années, mais qui existe déjà dans plusieurs pays européens. Le nombre d'entreprises se lançant dans l'aventure au niveau européen est cependant encore assez limité : les usages locaux et les mécanismes du choix des entreprises, en Italie ou en Espagne, par exemple, ne laissent guère de place à une concurrence internationale. Mais d'autres formes d'intervention sont possibles. L'Inrap a ainsi multiplié les actions de coopération institutionnelle en matière de conseil juridique, organisationnel et technique auprès de services d'archéologie préventive naissants (Albanie, bientôt Tunisie), ou dans le cadre d'opérations d'aménagement (diagnostic sur l'espace de la station du métro d'Alger, fouille du site de Moulay Idriss sur le tronçon marocain de l'autoroute trans-maghrébine ou, en coopération avec l'autorité israélienne des Antiquités, sur les sites de Beisamoun et de Césarée en Israël). L'objectif de cette coopération est de promouvoir une certaine idée de la constitution de structures d'archéologie préventive dans les pays ayant ratifié, ou non, la convention de Malte. Le modèle proposé est alors plutôt celui 
de l'intégration cohérente de l'ensemble de la chaîne de traitement des données au sein d'un seul organisme d'archéologie préventive pratiquant le terrain, l'élaboration des données et la recherche qu'elles nécessitent.

Certains pays, sans avoir signé la convention de Malte, ont inauguré une politique allant dans le même sens, telle la Turquie, qui a lancé sous la direction du musée d'Istanbul un gigantesque chantier d'archéologie urbaine à la sortie du tunnel qui passera sous le Bosphore, intégrant la conservation et l'exploitation scientifique des données.

Mais les différences de conception du travail d'archéologie préventive s'accentuent, et nous le voyons se développer actuellement en France, entre une organisation intégrée comme celle de l'Inrap, et une organisation morcelée (dominante en Espagne, Italie ou Grande-Bretagne) dans laquelle on demande aux sociétés d' « archéologie commerciale » de ne fournir qu'une partie du traitement de l'information archéologique primaire issue du terrain. Il est évident que ces sociétés, vu l'état d'esprit « libéral » de l'époque, pourraient sous peu chercher à élargir l'espace du marché de l'archéologie et proposer leurs services autour de la Méditerranée, par exemple pour des projets d'aménagements. C'est déjà le cas dans le Golfe ou au Yémen. Par ailleurs, dans les pays qui ne souhaitent ou ne peuvent pas construire leurs propres services au sein des directions des Antiquités, le modèle de «l'archéologie commerciale» peut être un choix politique et économique.

Ce mode d'intervention et de contrat peut alors, comme en Europe, introduire une division institutionnelle du travail dans la chaîne de production des connaissances. Les pays acheteurs de services auront l'entière responsabilité de la conservation et de l'exploitation des données primaires produites dans les opérations préventives ou de sauvetage.

Nous sommes donc aujourd'hui à un tournant majeur des conditions de production du savoir archéologique. Selon les types d'organisation choisis dans les pays dont nous sommes les partenaires, le mode et les points focaux de la coopération devront être profondément adaptés pour limiter la perte probable d'information et de connaissance.

\section{Quelques questions majeures pour l'avenir de l'archéologie.}

1 - La France, comme d'autres pays, a un problème d'articulation entre sa stratégie archéologique dans l'Hexagone et sa stratégie à l'étranger. Il serait bon de faire émerger une politique européenne d'accords et d'interventions entre les pays européens. L'extension de l'Union européenne en Europe centrale rend cette dimension de plus en plus souhaitable, mais il est anormal et décalé aujourd'hui que les grands pays d'Europe occidentale marchent chacun d'un pas différent, ce qui encourage des politiques régionales qui risquent de morceler davantage l'espace européen. Au contraire, sur la base d'une véritable politique européenne de l'archéologie, les régions pourraient toutes trouver leur place dans un cadre unifié et avec une relative autonomie d'intervention. Le nouveau décret de la Région Sicile du 28 juin 2010, qui réorganise la gestion des biens culturels dans lîle (dotée d'autonomie) par la création d'une multitude de lieux de pouvoir favorisant une «identité sicilienne », est un exemple porteur d'effets pervers et qu'il ne faudrait pas suivre aveuglément sous peine de parvenir rapidement à une déstructuration des territoires. De son côté, l'Association européenne des archéologues travaille depuis des années à faire émerger une coordination des politiques archéologiques dans l'Union.

2 - Cette question doit être de plus en plus l'objet de discussions avec nos partenaires extraeuropéens (Maghreb, Proche et Moyen-Orient, Extrême-Orient, Amériques, Océanie), afin de leur montrer que le temps des missions strictement nationales, héritières de la phase coloniale, doit être remplacé par celui de partenariats plus larges engageant plusieurs pays européens dans la même mission et sur le même site. Il est paradoxal aujourd'hui de constater que les Français, les Italiens, les Espagnols, les Allemands et les Britanniques, qui collaborent de plus en plus souvent entre eux, se séparent chacun derrière leur drapeau dès qu'ils sont sur la rive sud de la Méditerranée ou dans des pays plus lointains. Il est encore plus dommage de voir des partenaires être tentés de se diviser au moment d'aller travailler ailleurs, rompant ainsi les logiques de travail conjoint qui se mettent en place en Europe. Tous ont intérêt à modifier leurs points de vue et pas seulement pour bénéficier des crédits européens.

3 - Les articulations et les passerelles entre archéologie hexagonale et archéologie à l'étranger doivent se multiplier. Il est en effet regrettable que les archéologues français soient le plus souvent engagés dans un seul de ces deux domaines complémentaires, et se désintéressent complètement de l'autre comme s'il s'agissait d'un autre métier. Les archéologues travaillant en France ont besoin d'expériences internationales qui les confrontent à de nouvelles traditions de recherche et à d'autres questionnements. Certes, certains l'ont déjà fait mais ils restent trop souvent des cas isolés. La commission consultative pour les recherches archéologiques à l'étranger du ministère des Affaires étrangères et européennes (MAEE) a récemment vu avec intérêt des demandes convaincantes de ce point de vue. Il est fondamental que ce mixage s'opère face à ce qu'il faut bien appeler une hémorragie des chefs de mission vers l'étranger dans certains secteurs géographiques (Maghreb, Espagne, etc.) avec la multiplication des départs en retraite.

4- Une autre articulation défaillante concerne les missions et les instituts implantés à l'étranger, qu'ils relèvent du MAEE ou du MEFr (les cinq Écoles françaises à l'étranger). Dans ce domaine, il faut éviter que les instituts soient des relais exclusifs qui pourraient parfois faire écran à des relations bilatérales multiples. Mais ils ne doivent pas non plus être déconnectés du dispositif 

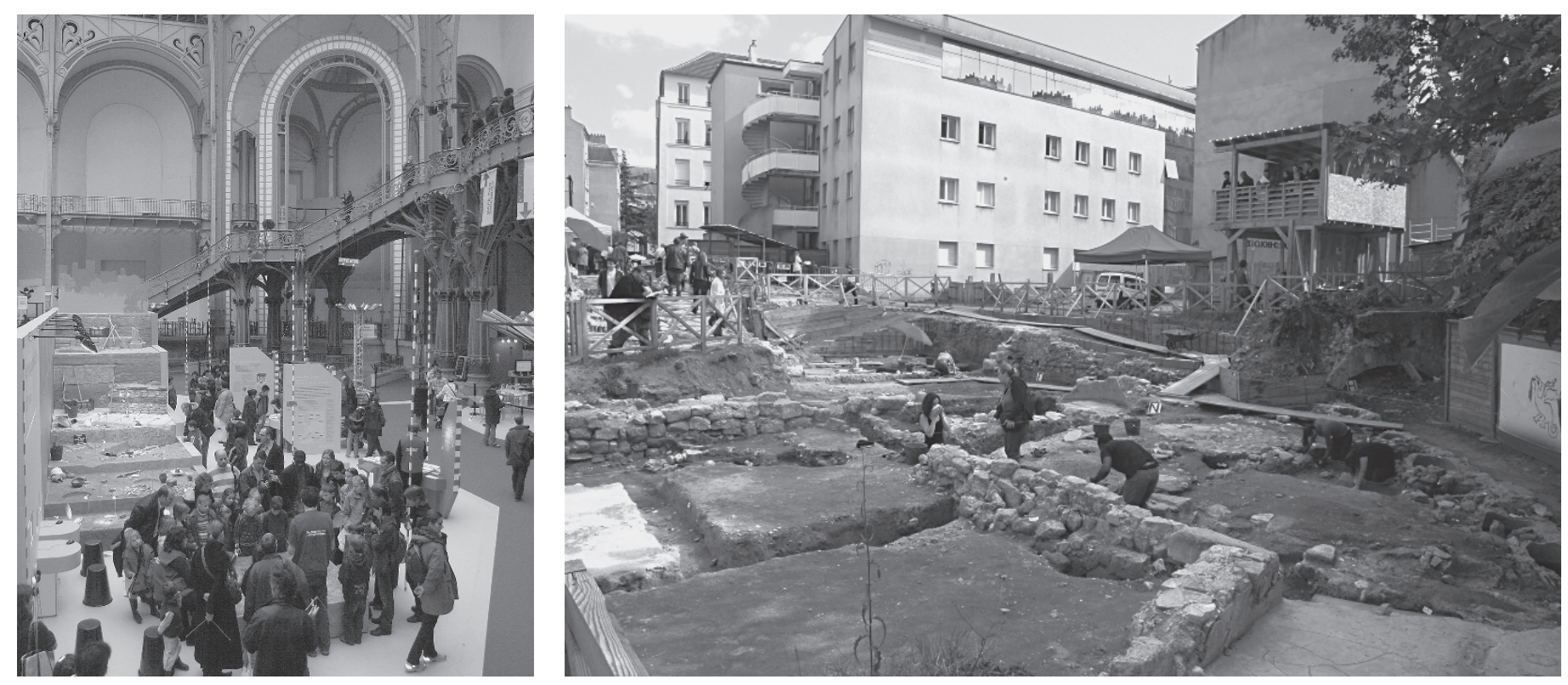

\begin{tabular}{|l|l|}
\hline \multicolumn{1}{|c|}{$\begin{array}{c}\text { Diplôme universitaire } \\
\text { minimum requis }\end{array}$} & \multicolumn{1}{c|}{ Pays } \\
\hline $\begin{array}{l}\text { Licence en archéologie } \\
\text { (ou spécialisation) }\end{array}$ & Belgique, Chypre, Espagne, Grèce, \\
\hline Master en archéologie & $\begin{array}{l}\text { Allemagne, Autriche, Hongrie, } \\
\text { Pays-Bas, Pologne, République } \\
\text { Tchèque, Slovaquie, Slovénie }\end{array}$ \\
\hline Pas de diplôme requis & Irlande, Royaume-Uni \\
\hline
\end{tabular}

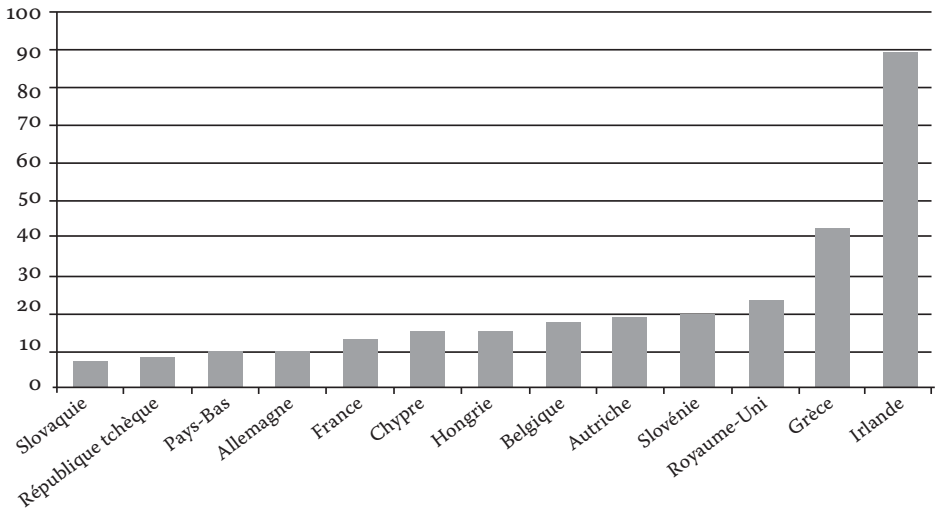

Nombre d'archéologues pour 100 ooo hectares (2008)

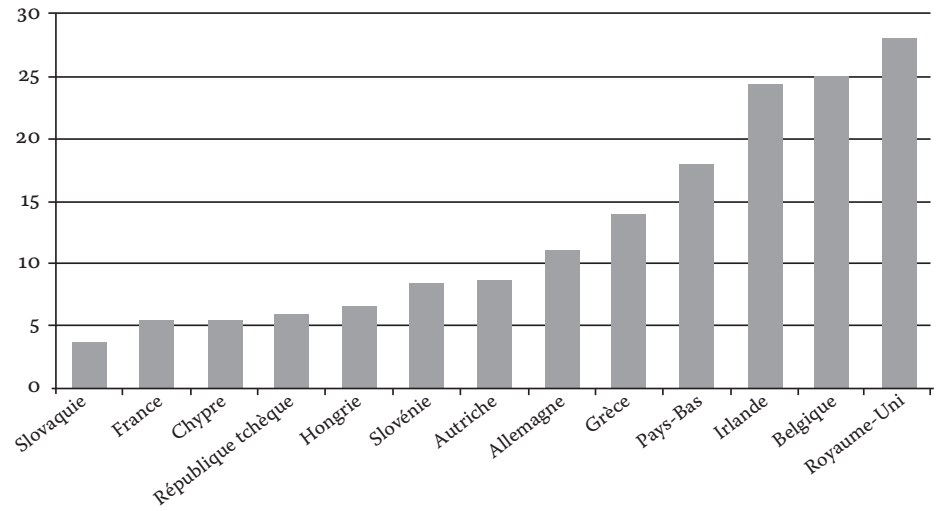

[Fig.1] Coupe stratigraphique présentée à la Ville européenne des sciences, Grand Palais, 2008.

[Fig.2] Fouille de l'îlot du Cygne à Saint-Denis, en Seine-Saint-Denis.

[Fig.3] Ce qui frappe immédiatement c'est la très forte proportion d'archéologues en Irlande (89 pour 100 ooo actifs). Cela s'explique clairement par l'afflux massif de professionnels venus de nombreux pays européens (Pologne notamment) pour répondre à une très importante demande dans le secteur du patrimoine archéologique. Celle-ci était liée à l'explosion du volume des projets d'aménagement du territoire lié au « miracle » économique du «tigre celtique» et au rapide développement d'une archéologie commerciale. Depuis la récession mondiale de 2008 la situation a évolué dans un sens radicalement opposé... puisque près de $80 \%$ des archéologues irlandais ont perdu leur emplois! Pour la France, on peut remarquer que le nombre d'archéologues rapporté aux actifs du pays (env. 13 pour 100 ooo actifs) est loin d'être le plus élevé d'Europe et se situe même dans la frange inférieure. Au vu de la richesse patrimoniale de la France (tel qu'elle se reflète en termes de tourisme, pa exemple) on peut raisonnablement en conclure que cette proportion est trop basse. Cette conclusion est un élément de plus de la réflexion à mener sur les politiques à même de garantir la pleine application de la Convention de Malte sur notre territoire, y compris ses objectifs scientifiques et patrimoniaux.

[Fig.3] En corrélant le nombre d'archéologues à la superficie des pays on obtient une image de la densité de la profession par pays. Notons que parmi les quatre pays les plus denses se trouvent les Pays-Bas, l'Irlande et le Royaume-Uni, trois des pays où le système commercial de l'archéologie est devenu dominant. La multiplication d'offres d'entreprises commerciales, dont l'efficacité d'emploi reste à prouver, peut expliquer ce phénomène. En comparaison, les pays

traditionnellement orientés à une plus forte « présence » des services publics dans les questions d'archéologie (p. ex. la Grèce, la France) ont une densité moindre en archéologues. La France se trouve en effet en avant dernière position, ce qui confirme que le nombre d'archéologues sur le territoire national est encore insuffisant (en proportion) par rapport à nos voisins européens. 
des missions. Au cas par cas, on constate ici de fortes divergences qui ne tiennent le plus souvent qu'à une histoire particulière et au rôle de telle ou telle personnalité. Des conventions entre les instituts et les missions seraient souvent les bienvenues, y compris pour résoudre le difficile problème de la publication (pas forcément toujours sur papier) et de la diffusion des résultats. Il s'agit aussi d'articuler la recherche et la formation à la recherche, tout en évitant le concept ambigu et donc dangereux de « chantier-école» qui laisse, à juste titre, nos partenaires perplexes: comme si, pour la formation initiale de ses étudiants à l'archéologie, la France devait les envoyer s'exercer sur le patrimoine d'autres pays (le plus souvent extra-européens) alors qu'elle peut aussi bien les former en France à moindre coût. Bien évidemment les choses changent quand il s'agit de jeunes chercheurs engagés dans une thèse et qui ont, dès lors, un besoin indispensable de travail sur le terrain de leur sujet.

5 - Tout cela débouche sur des logiques de chaînes opératoires qui dépassent les clivages régionaux et qui devraient se mettre en place parallèlement à des réglementations coordonnées et cohérentes dans l'espace européen, ce qui n'est pas encore le cas. Cela permettrait des approches qui remettraient les territoires au centre de nos attentions. Territoires pris dans un sens de cohérence des écosystèmes, de l'histoire et des positionnements dans le monde d'aujourd'hui. L'archéologie est une discipline qui, par sa nature même, peut ambitionner de tenir le rôle de liant (sans impérialisme ni volonté de domination) dans une approche territoriale qui cesserait définitivement d'opposer le paysage au patrimoine tout en prenant en compte, dans le cadre du rassemblement des nécessaires compétences, toutes les facettes et toutes les potentialités d'un espace où ont vécu les hommes d'hier et où vivent les hommes d'aujourd'hui, afin de préparer un avenir plus serein pour les hommes de demain.

\section{Archéologie dans l'Europe contemporaine (projet ACE) : perspectives européennes sur la médiation au public et la profession archéologique}

\author{
Kai Salas Rossenbach \\ Nathan Schlanger \\ Sonia Lévin \\ Coordination du projet ACE/Inrap
}

0 cience du passé, l'archéologie est aussi une discipline éminemment contemporaine, ancrée de multiples façons dans le présent. Dès ses origines, avec une accélération depuis la deuxième moitié $\mathrm{du} \mathrm{xx}^{\mathrm{e}}$ siècle, et plus encore durant ces 20 ou 30 dernières années, l'archéologie ne se limite pas au stéréotype d'un savoir érudit, distant et souvent confidentiel, et moins encore à une chasse au trésor plus ou moins systématique pour alimenter collections et musées. Au-delà de ses volets scientifiques et culturels, l'archéologie comprend aussi, dans sa constitution et dans sa pratique, des dimensions sociales, économiques et politiques. À la fois ressource et enjeu, elle est aujourd'hui présente - et le plus souvent bienvenue dans nos campagnes et dans nos villes, dans nos paysages quotidiens et nos destinations lointaines, dans nos politiques culturelles, scientifiques et patrimoniales, dans nos stratégies d'aménagement $\mathrm{du}$ territoire et de son occupation citoyenne. Car, enfin, c'est bien dans le présent que l'archéologie œuvre pour l'avenir du passé. Interrogeons cette contemporanéité : qu'en est-il de sa dimension internationale, européenne? Les mouvements et les accords internationaux ont souvent eu, on le sait, un effet de levier sur les législations et les pratiques nationales - telle la convention Unesco sur le Patrimoine mondial (1972), ou encore les Cconventions du Conseil de l'Europe pour la protection du patrimoine archéologique (Malte, 1992) et du paysage (Florence, 200o). Peut-on mettre en évidence ces incidences et les comparer? Peut-on identifier des pistes et des écueils, cerner des différences structurelles et des ambitions communes, pays par pays, à l'échelle de notre continent? C'est bien ce défi que l'Inrap a choisi de relever, dans le cadre de sa politique de développement et de collaboration internationale et en vertu de sa stature, étant l'un des premiers instituts de recherche et de protection du patrimoine archéologique à l'échelle européenne, sinon mondiale.

L'Inrap s'est ainsi associé à une douzaine de partenaires européens pour concevoir un projet pluriannuel de coopération transnationale dans le cadre du programme «Culture » de la Commission européenne (2007-2012) intitulé «Archaeology in Contemporary Europe: professional practices and public outreach» (cf. www.ace-archaeology.eu). Avec l'Inrap comme chef de file et signataire, en partenariat avec l'Agence exécutive «Éducation, Audiovisuel et Culture » (EACEA) de la Commission Européenne, AcE est un réseau de collaborations qui réunit et met en œuvre une gamme d'expériences et d'expertises, afin d'analyser et de mieux faire connaître les enjeux et les pratiques de l'archéologie dans l'Europe contemporaine. Réunis dans un esprit de complémentarité et de partage, les partenaires du projet représentent une gamme d'institutions de différents statuts et tailles - des organismes de service public, des universités, des centres de recherche, des associations, etc. provenant de plusieurs pays d'Europe de l'Ouest, de la Méditerranée et d'Europe centrale . Dans le cadre de ce partenariat, et suivant les intérêts et les spécificités de chacun, le projet se développe autour de plusieurs thématiques qui font l'objet de recherches collectives, de présentations et de publications. Parmi les pistes ouvertes, qui sont ici brièvement exposées, l'une porte sur des actions de médiation et de diffusion au public, et l'autre concerne plus spécifiquement la thématique de la profession archéologique. 